CUBO A Mathematical Journal

Vol.19, Noㅡ, (31-42). October 2017

\title{
On the solution set of a fractional integro-differential inclusion involving Caputo-Katugampola derivative
}

\author{
Aurelian Cernea \\ Faculty of Mathematics and Computer Science \\ University of Bucharest \\ Academiei 14, 010014 Bucharest, Romania \\ Academy of Romanian Scientists \\ Splaiul Independenţei 54, 050094 Bucharest, Romania \\ acernea@fmi.unibuc.ro
}

\begin{abstract}
We study an initial value problem associated to a fractional integro-differential inclusion defined by Caputo-Katugampola derivative and by a set-valued map with nonconvex values. We prove the arcwise connectedness of the solution set and that the set of selections corresponding to the solutions of the problem considered is a retract of the space of integrable functions on a given interval.
\end{abstract}

\section{RESUMEN}

Estudiamos un problema de valor inicial asociado a la inclusión íntegro-diferencial fraccionaria definida por la derivada de Caputo-Katugampola y por una aplicación multivaluada con valores no-convexos. Demostramos la arco-conexidad del conjunto solución y que el conjunto de selecciones correspondientes a las soluciones del problema considerado es un retracto del espacio de funciones integrables en un intervalo dado.

Keywords and Phrases: Differential inclusion, fractional derivative, initial value problem.

2010 AMS Mathematics Subject Classification: 34A60, 26A33, 34B15. 


\section{Introduction}

In the last years one may see a strong development of the theory of differential equations and inclusions of fractional order ([2,5,7-9] etc.). The main reason is that fractional differential equations are very useful tools in order to model many physical phenomena.

Recently, a generalized Caputo-Katugampola fractional derivative was proposed in [6] by Katugampola and afterwards he provided the existence of solutions for fractional differential equations defined by this derivative. This Caputo-Katugampola fractional derivative extends the well known Caputo and Caputo-Hadamard fractional derivatives. Also, in some recent papers [1,12], several qualitative properties of solutions of fractional differential equations defined by CaputoKatugampola derivative were obtained.

Differential inclusion is a generalization of the notion of ordinary differential equation that provides powerful tools for various fields of mathematical analysis. At the same time there are dynamics of economical, social and biological systems that are set-valued. Therefore, differential inclusions serve as natural models in such systems. An outstanding example comes from control theory. Namely, the equivalence between a control system and the corresponding differential inclusion, established by Filippov, allowed to obtained necessary and sufficient conditions of optimality using set-valued techniques.

In the present paper we study the following Cauchy problem

$$
\mathrm{D}_{\mathrm{c}}^{\alpha, \rho} \chi(\mathrm{t}) \in \mathrm{F}(\mathrm{t}, x(\mathrm{t}), \mathrm{V}(\mathrm{x})(\mathrm{t})) \text { a.e. }([0, \mathrm{~T}]), \quad x(0)=x_{0},
$$

where $\alpha \in(0,1], \rho>0, D_{c}^{\alpha, \rho}$ is the Caputo-Katugampola fractional derivative, $F:[0, T] \times \mathbf{R} \times \mathbf{R} \rightarrow$ $\mathcal{P}(\mathbf{R})$ is a set-valued map, $\mathrm{V}: \mathrm{C}([0, \mathrm{~T}], \mathbf{R}) \rightarrow \mathrm{C}([0, \mathrm{~T}], \mathbf{R})$ is a nonlinear Volterra integral operator defined by $\mathrm{V}(\mathrm{x})(\mathrm{t})=\int_{0}^{\mathrm{t}} \mathrm{k}(\mathrm{t}, \mathrm{s}, x(\mathrm{~s}))$ ds with $\mathrm{k}(., . .):.[0, \mathrm{~T}] \times \mathbf{R} \times \mathbf{R} \rightarrow \mathbf{R}$ a given function and $x_{0} \in \mathbf{R}$.

Our goal is twofold. On one hand, we prove the arcwise connectedness of the solution set of problem (1.1) when the set-valued map is Lipschitz in the second and third variable. On the other hand, under such type of hypotheses on the set-valued map we establish a more general topological property of the solution set of problem (1.1). Namely, we prove that the set of selections of the set-valued map $F$ that correspond to the solutions of problem (1.1) is a retract of $L^{1}([0, T], \mathbf{R})$. Both results are essentially based on Bressan and Colombo results ([3]) concerning the existence of continuous selections of lower semicontinuous multifunctions with decomposable values.

In the theory of ordinary differential equations Kneser's theorem states that the solution set of an ordinary differential equation is connected, i.e., it cannot be represented as a union of two closed sets without common points. In the case of differential inclusions, although the solution set multifunction is not, in general, convex valued we are able to prove its arcwise connectedness and therefore, our result may be regarded as an extension of the classical theorem of Kneser.

We note that similar results for fractional differential inclusions defined by classical Caputo fractional derivative are obtained in our previous paper [4]. The results in [4] extend to the case of 
fractional differential inclusions the results in $[10,11]$ obtained for ordinary differential inclusions. The present paper generalizes and unifies all these results in the case of the more general problem (1.1).

The paper is organized as follows: in Section 2 we present the notations, definitions and the preliminary results to be used in the sequel and in Section 3 we prove our main results.

\section{Preliminaries}

Let $\mathrm{T}>0, \mathrm{I}:=[0, \mathrm{~T}]$ and denote by $\mathcal{L}(\mathrm{I})$ the $\sigma$-algebra of all Lebesgue measurable subsets of $\mathrm{I}$. Let $X$ be a real separable Banach space with the norm |.|. Denote by $\mathcal{P}(X)$ the family of all nonempty subsets of $X$ and by $\mathcal{B}(X)$ the family of all Borel subsets of $X$. If $A \subset I$ then $\chi_{A}():. I \rightarrow\{0,1\}$ denotes the characteristic function of $A$. For any subset $A \subset X$ we denote by $\operatorname{cl}(A)$ the closure of A.

The distance between a point $x \in X$ and a subset $A \subset X$ is defined as usual by $d(x, A)=$ $\inf \{|x-a| ; a \in A\}$. We recall that Pompeiu-Hausdorff distance between the closed subsets $A, B \subset X$ is defined by $d_{H}(A, B)=\max \left\{d^{*}(A, B), d^{*}(B, A)\right\}, d^{*}(A, B)=\sup \{d(a, B) ; a \in A\}$.

As usual, we denote by $\mathrm{C}(\mathrm{I}, \mathrm{X})$ the Banach space of all continuous functions $\mathrm{X}: \mathrm{I} \rightarrow \mathrm{X}$ endowed with the norm $|x|_{C}=\sup _{t \in I}|x(t)|$ and by $L^{1}(I, X)$ the Banach space of all (Bochner) integrable functions $x: I \rightarrow X$ endowed with the norm $|x|_{1}=\int_{0}^{T}|x(t)| d t$.

We recall first several preliminary results we shall use in the sequel.

A subset $D \subset L^{1}(I, X)$ is said to be decomposable if for any $u, v \in D$ and any subset $A \in \mathcal{L}(\mathrm{I})$ one has $u \chi_{A}+v \chi_{B} \in D$, where $B=I \backslash A$.

We denote by $\mathcal{D}(\mathrm{I}, \mathrm{X})$ the family of all decomposable closed subsets of $\mathrm{L}^{1}(\mathrm{I}, \mathrm{X})$.

$\operatorname{Next}(S, d)$ is a separable metric space; we recall that a multifunction $G: S \rightarrow \mathcal{P}(X)$ is said to be lower semicontinuous (l.s.c.) if for any closed subset $C \subset X$, the subset $\{s \in S ; G(s) \subset C\}$ is closed. The next lemmas may be found in [3].

Lemma 2.1. If $\mathrm{F}: \mathrm{I} \rightarrow \mathcal{D}(\mathrm{I}, \mathrm{X})$ is a lower semicontinuous multifunction with closed nonempty and decomposable values then there exists $\mathrm{f}: \mathrm{I} \rightarrow \mathrm{L}^{1}(\mathrm{I}, \mathrm{X})$ a continuous selection from $F$.

Lemma 2.2. Let $\mathrm{G}(.,):. \mathrm{I} \times \mathrm{S} \rightarrow \mathcal{P}(\mathrm{X})$ be a closed-valued $\mathcal{L}(\mathrm{I}) \otimes \mathcal{B}(\mathrm{S})$-measurable multifunction such that $\mathrm{G}(\mathrm{t},$.$) is l.s.c. for any \mathrm{t} \in \mathrm{I}$.

Then the multifunction $\mathrm{G}^{*}():. \mathrm{S} \rightarrow \mathcal{D}(\mathrm{I}, \mathrm{X})$ defined by

$$
\mathrm{G}^{*}(\mathrm{~s})=\left\{\mathrm{f} \in \mathrm{L}^{1}(\mathrm{I}, \mathrm{X}) ; \quad \mathrm{f}(\mathrm{t}) \in \mathrm{G}(\mathrm{t}, \mathrm{s}) \text { a.e. }(\mathrm{I})\right\}
$$

is l.s.c. with nonempty closed values if and only if there exists a continuous mapping $\mathrm{q}():. \mathrm{S} \rightarrow$ $\mathrm{L}^{1}(\mathrm{I}, \mathrm{X})$ such that

$$
\mathrm{d}(0, \mathrm{G}(\mathrm{t}, \mathrm{s})) \leq \mathrm{q}(\mathrm{s})(\mathrm{t}) \text { a.e. }(\mathrm{I}), \forall \mathrm{s} \in \mathrm{S} .
$$


Lemma 2.3. Let $\mathrm{H}():. S \rightarrow \mathcal{D}(\mathrm{I}, \mathrm{X})$ be a l.s.c. multifunction with closed decomposable values and let $\mathrm{a}():. \mathrm{S} \rightarrow \mathrm{L}^{1}(\mathrm{I}, \mathrm{X}), \mathrm{b}():. \mathrm{S} \rightarrow \mathrm{L}^{1}(\mathrm{I}, \mathbf{R})$ be continuous such that the multifunction $\mathrm{F}():. \mathrm{S} \rightarrow \mathcal{D}(\mathrm{I}, \mathrm{X})$ defined by

$$
\mathrm{F}(\mathrm{s})=\operatorname{cl}\{\mathrm{f} \in \mathrm{H}(\mathrm{s}) ; \quad|\mathrm{f}(\mathrm{t})-\mathrm{a}(\mathrm{s})(\mathrm{t})|<\mathrm{b}(\mathrm{s})(\mathrm{t}) \quad \text { a.e. }(\mathrm{I})\}
$$

has nonempty values.

Then $\mathrm{F}($.$) has a continuous selection.$

Let $\rho>0$.

Definition 2.4. ([6]) a) The generalized left-sided fractional integral of order $\alpha>0$ of a Lebesgue integrable function $f:[0, \infty) \rightarrow \mathbf{R}$ is defined by

$$
I^{\alpha, \rho} f(t)=\frac{\rho^{1-\alpha}}{\Gamma(\alpha)} \int_{0}^{t}\left(t^{\rho}-s^{\rho}\right)^{\alpha-1} s^{\rho-1} f(s) d s
$$

provided the right-hand side is pointwise defined on $(0, \infty)$ and $\Gamma($.$) is the (Euler's) Gamma function$ defined by $\Gamma(\alpha)=\int_{0}^{\infty} t^{\alpha-1} e^{-t} d t$.

b) The generalized fractional derivative, corresponding to the generalized left-sided fractional integral in (2.1) of a function $f:[0, \infty) \rightarrow \mathbf{R}$ is defined by

$$
D^{\alpha, \rho} f(t)=\left(t^{1-\rho} \frac{d}{d t}\right)^{n}\left(I^{n-\alpha, \rho}\right)(t)=\frac{\rho^{\alpha-n+1}}{\Gamma(n-\alpha)}\left(t^{1-\rho} \frac{d}{d t}\right)^{n} \int_{0}^{t} \frac{s^{\rho-1} f(s)}{\left(t^{\rho}-s^{\rho}\right)^{\alpha-n+1}} d s
$$

if the integral exists and $n=[\alpha]$.

c) The Caputo-Katugampola generalized fractional derivative is defined by

$$
D_{c}^{\alpha, \rho} f(t)=\left(D^{\alpha, \rho}\left[f(s)-\sum_{k=0}^{n-1} \frac{f^{(k)}(0)}{k !} s^{k}\right]\right)(t)
$$

We note that if $\rho=1$, the Caputo-Katugampola fractional derivative becames the well known Caputo fractional derivative. On the other hand, passing to the limit with $\rho \rightarrow 0+$, the above definition yields the Hadamard fractional derivative.

In what follows $\rho>0$ and $\alpha \in[0,1]$

Lemma 2.5. For a given integrable function $\mathrm{h}():.[0, \mathrm{~T}] \rightarrow \mathbf{R}$, the unique solution of the initial value problem

$$
D_{c}^{\alpha, \rho} x(t)=h(t) \quad \text { a.e. }([0, T]), \quad x(0)=x_{0}
$$

is given by

$$
x(t)=x_{0}+\frac{\rho^{1-\alpha}}{\Gamma(\alpha)} \int_{0}^{t}\left(t^{\rho}-s^{\rho}\right)^{\alpha-1} s^{\rho-1} h(s) d s
$$


For the proof of Lemma 2.2, see [6]; namely, Lemma 4.2.

A function $x \in C(I, R)$ is called a solution of problem (1.1) if there exists a function $f \in L^{1}(I, R)$ with $f(t) \in F(t, x(t), V(x)(t))$ a.e. (I) such that $D_{c}^{\alpha, \rho} x(t)=f(t)$ a.e. (I) and $x(0)=x_{0}$.

In this case $(\chi(),. f()$.$) is called a trajectory-selection pair of problem (1.1).$

We shall use the following notations for the solution sets and for the selection sets of problem $(1.1)$.

$$
\begin{gathered}
\mathcal{S}\left(x_{0}\right)=\{x \in \mathrm{C}(\mathrm{I}, \mathbf{R}) ; \quad x \text { is a solution of }(1.1)\}, \\
\tilde{f}(\mathrm{t})=x_{0}+\frac{\rho^{1-\alpha}}{\Gamma(\alpha)} \int_{0}^{t}\left(t^{\rho}-s^{\rho}\right)^{\alpha-1} s^{\rho-1} f(s) d s, \\
\mathcal{T}\left(x_{0}\right)=\left\{f \in L^{1}(I, \mathbf{R}) ; \quad f(t) \in \mathrm{F}(\mathrm{t}, \tilde{\mathrm{f}}(\mathrm{t}), \mathrm{V}(\tilde{\mathrm{f}})(\mathrm{t})) \text { a.e. } \mathrm{I}\right\} .
\end{gathered}
$$

\section{The main results}

In order to prove our topological properties of the solution set of problem (1.1) we need the following hypotheses.

Hypothesis 3.1. i) $\mathrm{F}(.$, .) $: \mathrm{I} \times \mathbf{R} \times \mathbf{R} \rightarrow \mathcal{P}(\mathbf{R})$ has nonempty closed values and is $\mathcal{L}(\mathrm{I}) \otimes$ $\mathcal{B}(\mathbf{R} \times \mathbf{R})$ measurable.

ii) There exists $\mathrm{L}(.) \in \mathrm{L}^{1}(\mathrm{I},(0, \infty))$ such that, for almost all $\mathrm{t} \in \mathrm{I}, \mathrm{F}(\mathrm{t}, . .$.$) is \mathrm{L}(\mathrm{t})$-Lipschitz in the sense that

$$
\mathrm{d}_{\mathrm{H}}\left(\mathrm{F}\left(\mathrm{t}, \mathrm{x}_{1}, \mathrm{y}_{1}\right), \mathrm{F}\left(\mathrm{t}, \mathrm{x}_{2}, \mathrm{y}_{2}\right)\right) \leq \mathrm{L}(\mathrm{t})\left(\left|\mathrm{x}_{1}-\mathrm{x}_{2}\right|+\left|\mathrm{y}_{1}-\mathrm{y}_{2}\right|\right) \quad \forall \mathrm{x}_{1}, \mathrm{x}_{2}, \mathrm{y}_{1}, \mathrm{y}_{2} \in \mathbf{R} .
$$

iii) There exists $p \in \mathrm{L}^{1}(\mathrm{I}, \mathbf{R})$ such that

$$
d_{H}(\{0\}, F(t, 0, V(0)(t))) \leq p(t) \text { a.e. I. }
$$

iv) $\mathrm{k}(., .,):. \mathrm{I} \times \mathbf{R} \times \mathbf{R} \rightarrow \mathbf{R}$ is a function such that $\forall x \in \mathbf{R},(\mathrm{t}, \mathrm{s}) \rightarrow \mathrm{k}(\mathrm{t}, \mathrm{s}, \mathrm{x})$ is measurable.

v) $|k(t, s, x)-k(t, s, y)| \leq L(t)|x-y| \quad$ a.e. $(t, s) \in I \times I, \quad \forall x, y \in \mathbf{R}$.

We use next the following notations

$$
M(t):=L(t)\left(1+\int_{0}^{t} L(u) d u\right), \quad t \in I, \quad I^{\alpha, \rho} M:=\sup _{t \in I}\left|I^{\alpha, \rho} M(t)\right| .
$$

Theorem 3.2. Assume that Hypothesis 3.1 is satisfied and $\mathrm{I}^{\alpha, \rho} \mathrm{M}<1$.

Then for any $\xi_{0} \in \mathbf{R}$ the solution set $\mathcal{S}\left(\xi_{0}\right)$ is arcwise connected in the space $\mathrm{C}(\mathrm{I}, \mathbf{R})$.

Proof. Let $\xi_{0} \in \mathbf{R}$ and $x_{0}, x_{1} \in \mathcal{S}\left(\xi_{0}\right)$. Therefore there exist $f_{0}, f_{1} \in L^{1}(I, R)$ such that $x_{0}(t)=\xi_{0}+\frac{\rho^{1-\alpha}}{\Gamma(\alpha)} \int_{0}^{t}\left(t^{\rho}-u^{\rho}\right)^{\alpha-1} u^{\rho-1} f_{0}(u) d u$ and $x_{1}(t)=\xi_{0}+\frac{\rho^{1-\alpha}}{\Gamma(\alpha)} \int_{0}^{t}\left(t^{\rho}-u^{\rho}\right)^{\alpha-1} u^{\rho-1} f_{1}(u) d u$, $\mathrm{t} \in \mathrm{I}$. 
For $\lambda \in[0,1]$ define

$$
x^{0}(\lambda)=(1-\lambda) x_{0}+\lambda x_{1} \text { and } g^{0}(\lambda)=(1-\lambda) f_{0}+\lambda f_{1}
$$

Obviously, the mapping $\lambda \mapsto \chi^{0}(\lambda)$ is continuous from $[0,1]$ into $C(I, \mathbf{R})$ and since $\mid g^{0}(\lambda)-$ $\left.g^{0}\left(\lambda_{0}\right)\right|_{1}=\left|\lambda-\lambda_{0}\right| \cdot\left|f_{0}-f_{1}\right|_{1}$ it follows that $\lambda \mapsto g^{0}(\lambda)$ is continuous from $[0,1]$ into $L^{1}(I, R)$.

Define the set-valued maps

$$
\begin{gathered}
\Psi^{1}(\lambda)=\left\{v \in \mathrm{L}^{1}(\mathrm{I}, \mathbf{R}) ; \quad v(\mathrm{t}) \in \mathrm{F}\left(\mathrm{t}, x^{0}(\lambda)(\mathrm{t}), \mathrm{V}\left(\mathrm{x}^{0}(\lambda)\right)(\mathrm{t})\right) \text { a.e. } \mathrm{I}\right\}, \\
\Phi^{1}(\lambda)= \begin{cases}\left\{\mathrm{f}_{0}\right\} & \text { if } \lambda=0 \\
\Psi^{1}(\lambda) & \text { if } 0<\lambda<1 \\
\left\{\mathrm{f}_{1}\right\} & \text { if } \lambda=1\end{cases}
\end{gathered}
$$

and note that $\Phi^{1}:[0,1] \rightarrow \mathcal{D}(\mathrm{I}, \mathbf{R})$ is lower semicontinuous. Indeed, let $C \subset \mathrm{L}^{1}(\mathrm{I}, \mathbf{R})$ be a closed subset, let $\left\{\lambda_{m}\right\}_{m \in \mathbf{N}}$ converges to some $\lambda_{0}$ and $\Phi^{1}\left(\lambda_{m}\right) \subset C$ for any $m \in \mathbf{N}$. Let $\nu_{0} \in \Phi^{1}\left(\lambda_{0}\right)$. Since the multifunction $t \mapsto F\left(t, x^{0}\left(\lambda_{m}\right)(t), V\left(x^{0}\left(\lambda_{m}\right)\right)(t)\right)$ is measurable, it admits a measurable selection $v_{\mathrm{m}}($.$) such that$

$$
\left|v_{m}(t)-v_{0}(t)\right|=d\left(v_{0}(t), F\left(t, x^{0}\left(\lambda_{m}\right)(t), V\left(x^{0}\left(\lambda_{m}\right)\right)(t)\right)\right. \text { a.e. I. }
$$

Taking into account Hypothesis 3.1 one may write

$$
\begin{gathered}
\left|v_{m}(t)-v_{0}(t)\right| \leq d_{H}\left(F\left(t, x^{0}\left(\lambda_{m}\right)(t), V\left(x^{0}\left(\lambda_{m}\right)\right)(t)\right), F\left(t, x^{0}\left(\lambda_{0}\right)(t),\right.\right. \\
\left.V\left(x^{0}\left(\lambda_{0}\right)\right)(t)\right) \leq L(t)\left[\left|x^{0}\left(\lambda_{m}\right)(t)-x^{0}\left(\lambda_{0}\right)(t)\right|+\int_{0}^{t} L(s) \mid x^{0}\left(\lambda_{m}\right)(s)-\right. \\
\left.x^{0}\left(\lambda_{0}\right)(s) \mid d s\right]=L(t)\left|\lambda_{m}-\lambda_{0}\right|\left[\left|x_{0}(t)-x_{1}(t)\right|+\int_{0}^{t} L(s)\left|x_{0}(s)-x_{1}(s)\right| d s\right]
\end{gathered}
$$

hence

$$
\left|v_{m}-v_{0}\right|_{1} \leq\left|\lambda_{m}-\lambda_{0}\right| \int_{0}^{T} L(t)\left[\left|x_{0}(t)-x_{1}(t)\right|+\int_{0}^{t} L(s)\left|x_{0}(s)-x_{1}(s)\right| d s\right] d t
$$

which implies that the sequence $v_{m}$ converges to $v_{0}$ in $L^{1}(I, R)$. Since $C$ is closed we infer that $v_{0} \in C$; hence $\Phi^{1}\left(\lambda_{0}\right) \subset C$ and $\Phi^{1}($.$) is lower semicontinuous.$

Next we use the following notation

$$
p_{0}(\lambda)(t)=\left|g^{0}(\lambda)(t)\right|+p(t)+L(t)\left(\left|x^{0}(\lambda)(t)\right|+\int_{0}^{t} L(s)\left|x^{0}(\lambda)(s)\right| d s\right),
$$

$\mathrm{t} \in \mathrm{I}, \lambda \in[0,1]$.

Since

$$
\begin{aligned}
& \left|p_{0}(\lambda)(t)-p_{0}\left(\lambda_{0}\right)(t)\right| \leq\left|\lambda-\lambda_{0}\right|\left[\left|f_{1}(t)-f_{0}(t)\right|+\right. \\
& \left.L(t)\left(\left|x_{0}(t)-x_{1}(t)\right|+\int_{0}^{t} L(s)\left|x_{0}(s)-x_{1}(s)\right| d s\right)\right]
\end{aligned}
$$


we deduce that $p_{0}($.$) is continuous from [0,1]$ to $L^{1}(I, \mathbf{R})$.

At the same time, from Hypothesis 3.1 it follows

$$
d\left(g^{0}(\lambda)(t), F\left(t, x^{0}(\lambda)(t), V\left(x^{0}(\lambda)\right)(t)\right) \leq p_{0}(\lambda)(t) \quad\right. \text { a.e. I. }
$$

Fix $\delta>0$ and for $\mathrm{m} \in \mathbf{N}$ we set $\delta_{\mathrm{m}}=\frac{\mathrm{m}+1}{\mathrm{~m}+2} \delta$.

We shall prove next that there exists a continuous mapping $g^{1}:[0,1] \rightarrow L^{1}(I, R)$ with the following properties

a) $g^{1}(\lambda)(t) \in F\left(t, x^{0}(\lambda)(t), V\left(x^{0}(\lambda)\right)(t)\right)$ a.e. $I$,

b) $g^{1}(0)=f_{0}, \quad g^{1}(1)=f_{1}$,

c) $\left|g^{1}(\lambda)(t)-g^{0}(\lambda)(t)\right| \leq p_{0}(\lambda)(t)+\delta_{0} \frac{\rho^{\alpha} \Gamma(\alpha+1)}{T^{\rho \alpha}}$ a.e. I.

Define

$$
G^{1}(\lambda)=\operatorname{cl}\left\{v \in \Phi^{1}(\lambda) ; \quad\left|v(t)-g^{0}(\lambda)(t)\right|<p_{0}(\lambda)(t)+\delta_{0} \frac{\rho^{\alpha} \Gamma(\alpha+1)}{T^{\rho \alpha}}, \quad \text { a.e. I }\right\}
$$

and, by (3.1), we find that $\mathrm{G}^{1}(\lambda)$ is nonempty for any $\lambda \in[0,1]$. Moreover, since the mapping $\lambda \mapsto p_{0}(\lambda)$ is continuous, we apply Lemma 2.3 and we obtain the existence of a continuous mapping $g^{1}:[0,1] \rightarrow \mathrm{L}^{1}(\mathrm{I}, \mathbf{R})$ such that $\mathrm{g}^{1}(\lambda) \in \mathrm{G}^{1}(\lambda) \forall \lambda \in[0,1]$, hence with properties a)-c).

Define now

$$
x^{1}(\lambda)(t)=\xi_{0}+\frac{\rho^{1-\alpha}}{\Gamma(\alpha)} \int_{0}^{t}\left(t^{\rho}-u^{\rho}\right)^{\alpha-1} u^{\rho-1} g^{1}(\lambda)(u) d u, \quad t \in I
$$

and note that, since $\left|\chi^{1}(\lambda)-\chi^{1}\left(\lambda_{0}\right)\right|_{C} \leq \frac{T^{\rho \alpha}}{\rho^{\alpha} \Gamma(\alpha+1)}\left|g^{1}(\lambda)-g^{1}\left(\lambda_{0}\right)\right|_{1}, \chi^{1}($.$) is continuous from [0,1]$ into $\mathrm{C}(\mathrm{I}, \mathbf{R})$.

Set $p_{m}(\lambda):=\left(I^{\alpha, \rho} M\right)^{m-1}\left(\frac{T^{\rho \alpha}}{\rho^{\alpha} \Gamma(\alpha+1)}\left|p_{0}(\lambda)\right|_{1}+\delta_{m}\right)$.

We shall prove that for all $m \geq 1$ and $\lambda \in[0,1]$ there exist $x^{m}(\lambda) \in C(I, R)$ and $g^{m}(\lambda) \in$ $\mathrm{L}^{1}(\mathrm{I}, \mathbf{R})$ with the following properties

i) $g^{\mathfrak{m}}(0)=f_{0}, \quad g^{\mathfrak{m}}(1)=f_{1}$,

ii) $g^{m}(\lambda)(t) \in F\left(t, x^{m-1}(\lambda)(t), V\left(x^{m-1}(\lambda)\right)(t)\right)$ a.e. I,

iii) $\mathrm{g}^{\mathrm{m}}:[0,1] \rightarrow \mathrm{L}^{1}(\mathrm{I}, \mathbf{R})$ is continuous,

iv) $\left|g^{1}(\lambda)(t)-g^{0}(\lambda)(t)\right| \leq p_{0}(\lambda)(t)+\delta_{0} \frac{\rho^{\alpha} \Gamma(\alpha+1)}{T^{\rho \alpha}}$,

v) $\left|g^{m}(\lambda)(t)-g^{m-1}(\lambda)(t)\right| \leq M(t) p_{m}(\lambda), \quad m \geq 2$,

vi) $x^{m}(\lambda)(t)=\xi_{0}+\frac{\rho^{1-\alpha}}{\Gamma(\alpha)} \int_{0}^{t}\left(t^{\rho}-u^{\rho}\right)^{\alpha-1} u^{\rho-1} g^{m}(\lambda)(u) d u, \quad t \in I$.

Assume that we have already constructed $g^{\mathrm{m}}($.$) and x^{\mathrm{m}}($.$) with i)-vi) and define$

$$
\Psi^{m+1}(\lambda)=\left\{v \in \mathrm{L}^{1}(\mathrm{I}, \mathbf{R}) ; \quad v(\mathrm{t}) \in \mathrm{F}\left(\mathrm{t}, \mathrm{x}^{\mathrm{m}}(\lambda)(\mathrm{t}), \mathrm{V}\left(\mathrm{x}^{\mathrm{m}}(\lambda)\right)(\mathrm{t})\right) \text { a.e. } \mathrm{I}\right\},
$$




$$
\Phi^{m+1}(\lambda)= \begin{cases}\left\{f_{0}\right\} & \text { if } \lambda=0 \\ \Psi^{m+1}(\lambda) & \text { if } 0<\lambda<1 \\ \left\{f_{1}\right\} & \text { if } \lambda=1\end{cases}
$$

As in the case $m=1$ we obtain that $\Phi^{m+1}:[0,1] \rightarrow \mathcal{D}(\mathrm{I}, \mathbf{R})$ is lower semicontinuous.

From ii), v) and Hypothesis 3.1, for almost all $t \in I$, we have

$$
\begin{gathered}
\left|x^{m}(\lambda)(t)-x^{m-1}(\lambda)(t)\right| \leq \frac{\rho^{1-\alpha}}{\Gamma(\alpha)} \int_{0}^{t}\left(t^{\rho}-u^{\rho}\right)^{\alpha-1} u^{\rho-1}\left|g^{m}(\lambda)(u)-g^{m-1}(\lambda)(u)\right| d u \leq \\
\frac{\rho^{1-\alpha}}{\Gamma(\alpha)} \int_{0}^{t}\left(t^{\rho}-u^{\rho}\right)^{\alpha-1} u^{\rho-1} M(u) p_{m}(\lambda) d u=I^{\alpha, \rho} M(t) p_{m}(\lambda) \leq I^{\alpha, \rho} M p_{m}(\lambda)<p_{m+1}(\lambda) .
\end{gathered}
$$

For $\lambda \in[0,1]$ consider the set

$$
\mathrm{G}^{\mathrm{m}+1}(\lambda)=\operatorname{cl}\left\{v \in \Phi^{\mathrm{m}+1}(\lambda) ; \quad\left|v(\mathrm{t})-\mathrm{g}^{\mathrm{m}}(\lambda)(\mathrm{t})\right|<\mathrm{M}(\mathrm{t}) \mathrm{p}_{\mathrm{m}+1}(\lambda) \quad \text { a.e. } \mathrm{I}\right\} .
$$

To prove that $G^{m+1}(\lambda)$ is not empty we note first that $r_{m}:=\left(I^{\alpha, \rho} M\right)^{m}\left(\delta_{m+1}-\delta_{m}\right)>0$ and by Hypothesis 3.1 and ii) one has

$$
\begin{gathered}
d\left(g^{m}(t), F\left(t, x^{m}(\lambda)(t), V\left(x^{m}(\lambda)\right)(t)\right) \leq L(t)\left(\left|x^{m}(\lambda)(t)-x^{m-1}(\lambda)(t)\right|+\right.\right. \\
\left.\int_{0}^{t} L(s)\left|x^{m}(\lambda)(s)-x^{m-1}(\lambda)(s)\right| d s\right) \leq L(t)\left(1+\int_{0}^{t} L(s) d s\right)\left|I^{\alpha, \rho} M(t)\right| p_{m}(\lambda) \\
=M(t)\left(p_{m+1}(\lambda)-r_{m}\right)<M(t) p_{m+1}(\lambda) .
\end{gathered}
$$

Moreover, since $\Phi^{\mathrm{m}+1}:[0,1] \rightarrow \mathcal{D}(\mathrm{I}, \mathbf{R})$ is lower semicontinuous and the maps $\lambda \rightarrow p_{m+1}(\lambda)$, $\lambda \rightarrow h^{\mathrm{m}}(\lambda)$ are continuous we apply Lemma 2.3 and we obtain the existence of a continuous selection $\mathrm{g}^{\mathrm{m}+1}$ of $\mathrm{G}^{\mathrm{m}+1}$.

Therefore,

$$
\left|x^{m}(\lambda)-x^{m-1}(\lambda)\right|_{C} \leq I^{\alpha, \rho} M p_{m}(\lambda) \leq\left(I^{\alpha, \rho} M\right)^{m}\left(\frac{T^{\rho \alpha}}{\rho^{\alpha} \Gamma(\alpha+1)}\left|p_{0}(\lambda)\right|_{1}+\delta\right)
$$

and thus $\left\{x^{\mathfrak{m}}(\lambda)\right\}_{\mathfrak{m} \in \mathbf{N}}$ is a Cauchy sequence in the Banach space $\mathrm{C}(\mathrm{I}, \mathbf{R})$, hence it converges to some function $x(\lambda) \in \mathrm{C}(\mathrm{I}, \mathbf{R})$.

Let $g(\lambda) \in \mathrm{L}^{1}(\mathrm{I}, \mathbf{R})$ be such that

$$
x(\lambda)(t)=\xi_{0}+\frac{\rho^{1-\alpha}}{\Gamma(\alpha)} \int_{0}^{t}\left(t^{\rho}-u^{\rho}\right)^{\alpha-1} u^{\rho-1} g(\lambda)(u) d u, \quad t \in I .
$$

The function $\lambda \mapsto \frac{T^{\rho \alpha}}{\rho^{\alpha} \Gamma(\alpha+1)}\left|p_{0}(\lambda)\right|_{1}+\delta$ is continuous, so it is locally bounded. Therefore the Cauchy condition is satisfied by $\left\{x^{\mathfrak{m}}(\lambda)\right\}_{\mathfrak{m} \in \mathbf{N}}$ locally uniformly with respect to $\lambda$ and this implies that the mapping $\lambda \rightarrow x(\lambda)$ is continuous from $[0,1]$ into $C(I, \mathbf{R})$. Obviously, the convergence of the sequence $\left\{x^{\mathrm{m}}(\lambda)\right\}$ to $x(\lambda)$ in $C(I, \mathbf{R})$ implies that $g^{\mathrm{m}}(\lambda)$ converges to $g(\lambda)$ in $\mathrm{L}^{1}(\mathrm{I}, \mathbf{R})$. 
Finally, from ii), Hypothesis 3.1 and from the fact that the values of $F$ are closed we obtain that $x(\lambda) \in \mathcal{S}\left(\xi_{0}\right)$. From i) and v) we have $x(0)=x_{0}, x(1)=x_{1}$ and the proof is complete.

In what follows we use the notations

$$
\tilde{u}(t)=x_{0}+\frac{\rho^{1-\alpha}}{\Gamma(\alpha)} \int_{0}^{t}\left(t^{\rho}-s^{\rho}\right)^{\alpha-1} s^{\rho-1} u(s) d s, \quad u \in L^{1}(I, R)
$$

and

$$
p_{0}(u)(t)=|u(t)|+p(t)+L(t)\left(|\tilde{u}(t)|+\int_{0}^{t} L(s)|\tilde{u}(s)| d s\right), \quad t \in I
$$

Let us note that

$$
d\left(u(t), F(t, \tilde{u}(t), V(\tilde{u})(t)) \leq p_{0}(u)(t) \quad \text { a.e. } I\right.
$$

and, since for any $\mathfrak{u}_{1}, \mathfrak{u}_{2} \in \mathrm{L}^{1}(\mathrm{I}, \mathbf{R})$

$$
\left|p_{0}\left(u_{1}\right)-p_{0}\left(u_{2}\right)\right|_{1} \leq\left(1+\left|I^{\alpha, \rho} M(T)\right|\right)\left|u_{1}-u_{2}\right|_{1}
$$

the mapping $\mathrm{p}_{0}: \mathrm{L}^{1}(\mathrm{I}, \mathbf{R}) \rightarrow \mathrm{L}^{1}(\mathrm{I}, \mathbf{R})$ is continuous.

Proposition 3.3. Assume that Hypothesis 3.1 is satisfied and let $\phi: \mathrm{L}^{1}(\mathrm{I}, \mathbf{R}) \rightarrow \mathrm{L}^{1}(\mathrm{I}, \mathbf{R})$ be a continuous map such that $\phi(\mathfrak{u})=\mathfrak{u}$ for all $\mathfrak{u} \in \mathcal{T}\left(\mathrm{x}_{0}\right)$. For $\mathfrak{u} \in \mathrm{L}^{1}(\mathrm{I}, \mathbf{R})$, we define

$$
\begin{gathered}
\Psi(\mathrm{u})=\left\{\mathrm{u} \in \mathrm{L}^{1}(\mathrm{I}, \mathbf{R}) ; \quad \mathrm{u}(\mathrm{t}) \in \mathrm{F}(\mathrm{t}, \widetilde{\phi(\mathrm{u})}(\mathrm{t}), \mathrm{V}(\widetilde{\phi(\mathrm{u})})(\mathrm{t})) \text { a.e. } \mathrm{I}\right\}, \\
\Phi(\mathrm{u})= \begin{cases}\{\mathrm{u}\} & \text { if } \mathrm{u} \in \mathcal{T}\left(\mathrm{x}_{0}\right), \\
\Psi(\mathrm{u}) & \text { otherwise. }\end{cases}
\end{gathered}
$$

Then the multifunction $\Phi: \mathrm{L}^{1}(\mathrm{I}, \mathbf{R}) \rightarrow \mathcal{P}\left(\mathrm{L}^{1}(\mathrm{I}, \mathbf{R})\right)$ is lower semicontinuous with closed decomposable and nonempty values.

The proof of Proposition 3.3 is similar to the proof of Proposition 3.2 in [4].

Theorem 3.4. Assume that Hypothesis 3.1 is satisfied, consider $\chi_{0} \in \mathbf{R}$ and assume $\mathrm{I}^{\alpha, \rho} \mathrm{M}<$ 1.

Then there exists a continuous mapping $\mathrm{g}: \mathrm{L}^{1}(\mathrm{I}, \mathbf{R}) \rightarrow \mathrm{L}^{1}(\mathrm{I}, \mathbf{R})$ such that

i) $\mathrm{g}(\mathrm{u}) \in \mathcal{T}\left(\mathrm{x}_{0}\right), \quad \forall \mathrm{u} \in \mathrm{L}^{1}(\mathrm{I}, \mathbf{R})$,

ii) $\mathrm{g}(\mathrm{u})=\mathrm{u}, \quad \forall \mathrm{u} \in \mathcal{T}\left(\mathrm{x}_{0}\right)$.

Proof. Fix $\delta>0$ and for $m \geq 0$ set $\delta_{\mathfrak{m}}=\frac{\mathfrak{m}+1}{\mathfrak{m}+2} \delta$ and define $p_{\mathfrak{m}}(u):=\left(I^{\alpha, \rho} M\right)^{m-1}\left(\frac{T^{\rho \alpha}}{\rho^{\alpha} \Gamma(\alpha+1)}\left|p_{0}(u)\right|_{1}+\right.$ $\left.\delta_{\mathrm{m}}\right)$, where $\tilde{u}$ and $p_{0}($.$) are defined in (3.2) and (3.3). By the continuity of the map p_{0}($.$) , already$ proved, we obtain that $p_{m}: L^{1}(I, R) \rightarrow L^{1}(I, R)$ is continuous.

We define $g_{0}(u)=u$ and we shall prove that for any $m \geq 1$ there exists a continuous map $g_{m}: L^{1}(I, R) \rightarrow L^{1}(I, R)$ that satisfies

$$
\mathrm{g}_{\mathrm{m}}(\mathrm{u})=\mathrm{u}, \quad \forall \mathrm{u} \in \mathcal{T}\left(\mathrm{x}_{0}\right),
$$


b)

$$
g_{m}(u)(t) \in F\left(t, \widetilde{g_{m-1}(u)}(t), V\left(\widetilde{g_{m-1}(u)}\right)(t)\right) \text { a.e. I, }
$$

c)

$$
\left|g_{1}(u)(t)-g_{0}(u)(t)\right| \leq p_{0}(u)(t)+\delta_{0} \frac{\rho^{\alpha} \Gamma(\alpha+1)}{T^{\rho \alpha}} \text { a.e. I, }
$$

$$
\left|g_{m}(u)(t)-g_{m-1}(t)\right| \leq M(t) p_{m}(u) \text { a.e. } I, m \geq 2 .
$$

For $u \in \mathrm{L}^{1}(\mathrm{I}, \mathbf{R})$, we define

$$
\begin{gathered}
\Psi_{1}(u)=\left\{v \in \mathrm{L}^{1}(\mathrm{I}, \mathbf{R}) ; \quad v(\mathrm{t}) \in \mathrm{F}(\mathrm{t}, \widetilde{\mathrm{u}}(\mathrm{t}), \mathrm{V}(\widetilde{\mathrm{u}})(\mathrm{t})) \text { a.e. } \mathrm{I}\right\}, \\
\Phi_{1}(\mathrm{u})= \begin{cases}\{\mathrm{u}\} & \text { if } \mathrm{u} \in \mathcal{T}\left(\mathrm{x}_{0}\right), \\
\Psi_{1}(\mathfrak{u}) & \text { otherwise }\end{cases}
\end{gathered}
$$

and by Proposition 3.3 (with $\phi(u)=u$ ) we obtain that $\Phi_{1}: \mathrm{L}^{1}(\mathrm{I}, \mathbf{R}) \rightarrow \mathcal{D}(\mathrm{I}, \mathbf{R})$ is lower semicontinuous. Moreover, due to (3.4), the set

$$
\mathrm{G}_{1}(\mathrm{u})=\operatorname{cl}\left\{v \in \Phi_{1}(\mathrm{u}) ; \quad|v(\mathrm{t})-\mathrm{u}(\mathrm{t})|<\mathrm{p}_{0}(\mathrm{u})(\mathrm{t})+\delta_{0} \frac{\rho^{\alpha} \Gamma(\alpha+1)}{T^{\rho \alpha}} \text { a.e. I }\right\}
$$

is not empty for any $u \in L^{1}(I, R)$. So applying Lemma 2.3, we find a continuous selection $g_{1}($.$) of$ $G_{1}($.$) that satisfies a)-c).$

Suppose we have already constructed $g_{i}(),. i=1, \ldots, m$ satisfying a)-d). For $u \in L^{1}(I, R)$ we define

$$
\begin{aligned}
& \Psi_{m+1}(u)=\left\{v \in L^{1}(I, R)\right.\left.v(t) \in F\left(t, \widetilde{g_{m}(u)}(t), V\left(\widetilde{g_{m}(u)}\right)(t)\right) \quad \text { a.e. } I\right\} \\
& \Phi_{m+1}(u)= \begin{cases}\{u\} & \text { if } u \in \mathcal{T}\left(x_{0}\right) \\
\Psi_{m+1}(u) & \text { otherwise. }\end{cases}
\end{aligned}
$$

We apply Proposition 3.3 (with $\phi(u)=g_{\mathfrak{m}}(u)$ ) and obtain that $\Phi_{\mathfrak{m}+1}($.$) is a lower semicontinuous$ multifunction with closed decomposable and nonempty values. Define the set

$$
\mathrm{G}_{\mathrm{m}+1}(\mathrm{u})=\operatorname{cl}\left\{v \in \Phi_{\mathrm{m}+1}(\mathrm{u}) ; \quad\left|v(\mathrm{t})-\mathrm{g}_{\mathrm{m}+1}(\mathrm{u})(\mathrm{t})\right|<\mathrm{M}(\mathrm{t}) \mathrm{p}_{\mathrm{m}+1}(\mathrm{u}) \text { a.e. I }\right\} .
$$

To prove that $G_{m+1}(u)$ is not empty we note first that $r_{m}:=\left(I^{\alpha, \rho} M\right)^{m}\left(\delta_{m+1}-\delta_{m}\right)>0$ and by Hypothesis 3.1 and b) one has

$$
\begin{aligned}
& d\left(g_{m}(t), F\left(t, \widetilde{g_{m}(u)}(t), V\left(\widetilde{g_{m}(u)}\right)(t)\right) \leq L(t)\left(\widetilde{\mid g_{m}(u)}(t)-\widetilde{g_{m-1}(u)}(t) \mid+\right.\right. \\
& \int_{0}^{t} L(s)\left|\widetilde{g_{m}(u)}(s)-\widetilde{g_{m-1}(u)}(s)\right| d s \leq M(t)\left(I^{\alpha, \rho} M\right) p_{m}(u)=M(t)\left(p_{m+1}(u)-r_{m}\right) \\
& <M(t) p_{m+1}(u) .
\end{aligned}
$$

Thus $G_{m+1}(u)$ is not empty for any $u \in L^{1}(I, R)$. With Lemma 2.3 , we find a continuous selection $g_{m+1}$ of $G_{m+1}$, satisfying a)-d). 
Therefore, we obtain that

$$
\left|g_{m+1}(u)-g_{m}(u)\right|_{1} \leq\left(I^{\alpha, \rho} M\right)^{m}\left(\frac{T^{\rho \alpha-1}}{\rho^{\alpha} \Gamma(\alpha+1)}\left|p_{0}(u)\right|_{1}+\delta\right)
$$

and this implies that the sequence $\left\{g_{\mathfrak{m}}(\mathfrak{u})\right\}_{\mathfrak{m} \in \mathbf{N}}$ is a Cauchy sequence in the Banach space $\mathrm{L}^{1}(\mathrm{I}, \mathbf{R})$. Let $g(u) \in L^{1}(I, R)$ be its limit. The function $u \rightarrow\left|p_{0}(u)\right|_{1}$ is continuous, hence it is locally bounded and the Cauchy condition is satisfied by $\left\{\boldsymbol{g}_{\mathfrak{m}}(\mathfrak{u})\right\}_{\mathfrak{m} \in \mathbf{N}}$ locally uniformly with respect to $u$. Hence the mapping $g():. \mathrm{L}^{1}(\mathrm{I}, \mathbf{R}) \rightarrow \mathrm{L}^{1}(\mathrm{I}, \mathbf{R})$ is continuous.

From a) it follows that $\mathrm{g}(\mathrm{u})=\mathrm{u}, \quad \forall \mathrm{u} \in \mathcal{T}\left(\mathrm{x}_{0}\right)$ and from $\mathrm{b}$ ) and the fact that $\mathrm{F}$ has closed values we obtain that

$$
g(u)(t) \in F(t, \widetilde{g(u)}(t), V(\widetilde{g(u)})(t)) \quad \text { a.e. I } \quad \forall u \in L^{1}(I, R) .
$$

and the proof is complete.

Remark 3.5. We recall that if $Y$ is a Hausdorff topological space, a subspace $X$ of $Y$ is called retract of $Y$ if there is a continuous map $h: Y \rightarrow X$ such that $h(x)=x, \forall x \in X$.

Therefore, by Theorem 3.4, for any $x_{0} \in \mathbf{R}$, the set $\mathcal{T}\left(x_{0}\right)$ of selections of solutions of (1.1) is a retract of the Banach space $L^{1}(I, \mathbf{R})$.

Example 3.6. Consider $\alpha=\frac{1}{2}, \rho=1, T=1, x_{0}=1$ and $c<\min \left\{1, \frac{3}{6+2 \Gamma\left(\frac{1}{2}\right)}\right\}$. Define $\mathrm{F}(.,):. \mathrm{I} \times \mathbf{R} \times \mathbf{R} \rightarrow \mathcal{P}(\mathbf{R})$ by

$$
F(t, x, y)=\left[-a \frac{|x|}{1+|x|}, 0\right] \cup\left[0, a \frac{|y|}{1+|y|}\right], \quad a=c \Gamma\left(\frac{1}{2}\right)
$$

and $k(., .,):. I \times \mathbf{R} \times \mathbf{R} \rightarrow \mathbf{R}$ by $k(t, s, x)=a x$.

Since

$$
\begin{gathered}
\sup \{|u| ; \quad u \in F(t, x, y)\} \leq a \quad \forall t \in[0,1], x, y \in \mathbf{R}, \\
d_{H}\left(F\left(t, x_{1}, y_{1}\right), F\left(t, x_{2}, y_{2}\right)\right) \leq a\left|x_{1}-x_{2}\right|+a\left|y_{1}-y_{2}\right| \quad \forall x_{1}, x_{2}, y_{1}, y_{2} \in \mathbf{R},
\end{gathered}
$$

in this case $\mathrm{p}(\mathrm{t}) \equiv \mathrm{a}, \mathrm{L}(\mathrm{t}) \equiv \mathrm{a}, \mathrm{M}(\mathrm{t})=\mathrm{a}(1+\mathrm{at})$ and, taking into account the choice of $\mathrm{c}$,

$$
\mathrm{I}^{\frac{1}{2}, 1} \mathrm{M}(\mathrm{t})=2 \mathrm{ct} \mathrm{t}^{1 / 2}\left[1+\frac{2 \mathrm{c} \Gamma\left(\frac{1}{2}\right)}{3} \mathrm{t}\right] \leq 2 \mathrm{c}\left[1+\frac{2 \mathrm{c} \Gamma\left(\frac{1}{2}\right)}{3}\right]<1 \quad \forall \mathrm{t} \in[0,1] .
$$

Therefore, applying Theorems 3.2 and 3.4 to the problem

$$
D_{c}^{\frac{1}{2}, 1} x(t) \in\left[-a \frac{|x(t)|}{1+|x(t)|}, 0\right] \cup\left[0, a^{2} \frac{\left|\int_{0}^{t} x(s) d s\right|}{1+a\left|\int_{0}^{t} x(s) d s\right|}\right], \quad x(0)=1,
$$

we deduce that its solution set $\mathcal{S}(1)$ is arcwise connected in the space $\mathrm{C}([0,1]$, $\mathbf{R})$ and its set of selections of solutions $\mathcal{T}(1)$ is a retract of the Banach space $\mathrm{L}^{1}([0,1], \mathbf{R})$. 


\section{References}

[1] R. Almeida, A. B. Malinowski and T. Odzijewicz, Fractional differential equations with dependence on the Caputo-Katugampola derivative, J. Comput. Nonlin. Dyn., 11 (2016), ID 061017, 11 pp.

[2] D. Băleanu, K. Diethelm, E. Scalas and J. J. Trujillo, Fractional Calculus Models and Numerical Methods, World Scientific, Singapore, 2012.

[3] A. Bressan and G. Colombo, Extensions and selections of maps with decomposable values, Studia Math., 90 (1988), 69-86.

[4] A. Cernea, On a fractional integrodifferential inclusion, Electronic J. Qual. Theory Differ. Equ., 2014 (2014), no. 25, 1-11.

[5] K. Diethelm, The Analysis of Fractional Differential Equations, Springer, Berlin, 2010.

[6] U. N. Katugampola, A new approach to generalized fractional derivative, Bull. Math. Anal. Appl., 6 (2014) 1-15.

[7] A. Kilbas, H.M. Srivastava and J.J. Trujillo, Theory and Applications of Fractional Differential Equations, Elsevier, Amsterdam, 2006.

[8] K. Miller and B. Ross, An Introduction to the Fractional Calculus and Differential Equations, John Wiley, New York, 1993.

[9] I. Podlubny, Fractional Differential Equations, Academic Press, San Diego,1999.

[10] V. Staicu, On the solution sets to differential inclusions on unbounded interval, Proc. Edinburgh Math. Soc., 43 (2000), 475-484.

[11] V. Staicu, Arcwise conectedness of solution sets to differential inclusions, J. Math. Sciences 120 (2004), 1006-1015.

[12] S. Zeng, D. Băleanu, Y. Bai, G. Wu, Fractional differential equations of Caputo-Katugampola type and numerical solutions, Appl. Math. Comput., 315 (2017), 549-554. 